\title{
Ant Colony Optimization based Optimal Power Flow Analysis for the Iraqi Super High Voltage Grid
}

\author{
Firas M. Tuaimah \\ University of Baghdad \\ Baghdad, Iraq
}

\author{
Yaser Nadhum Abd \\ Iraqi Operation and Control Office \\ Baghdad, Iraq
}

\author{
Fahad A. Hameed \\ University of Baghdad \\ Diwaniyah, Iraq
}

\begin{abstract}
In this paper, the Ant Colony Optimization (ACO) based Optimal Power Flow (OPF) analysis implemented using MATLAB ${ }^{\circledR}$ is applied for the Iraqi Super High (SHV) grid, which consists of (11) generation and (13) load bus connected to each other with $400-\mathrm{kV}$ power transmission lines. The results obtained with the proposed approach are presented and compared favorably with results of other approaches, like the Linear Programming (LP) method.

All data used this analysis is taken from the Iraqi Operation and Control Office, which belongs to the ministry of electricity.
\end{abstract}

\section{Keywords}

Optimal Power Flow, Ant Colony Optimization (ACO), Active power dispatch.

\section{INTRODUCTION}

The optimal power flow (OPF) problem was introduced by Carpentier [1] in 1962 as a network constrained economic dispatch problem. Since then, the OFF problem has been intensively studied and widely used in power system operation and planning[2]. The OPF problem aims to achieve an optimal solution of a specific power system objective function, such as fuel cost, by adjusting the power system control variables, while satisfying a set of operational and physical constraints. The control variables include the generator real powers, the generator bus voltages, the tap ratios of transformer and the reactive power generations of VAR sources. State variables are slack bus power, load bus voltages, generator reactive power outputs, and network power flows. The constrains include inequality ones which are the limits of control variables and state variables; and equality ones which are the power flow equations. The OPF problem can be formulated as a nonlinear constrained optimization problem.

The first methods used to solve this kind of optimization problems, such as Linear Programming (LP); Non-Linear Programming (NLP); Mixed-Integer Programming (MIP); Newton method; and Interior Point method [3-4] were introduced by operational research. These techniques are literature-known as the traditional techniques.

In the last two decades the use of techniques based on Artificial Intelligence (AI) has increased in order to overcome some difficulties of the traditional ones, i.e. metaheuristics based on biological processes which are able to find a good solution in less time when compared with traditional techniques are being increasingly used. The advantage of metaheuristcs based methods is their faster performance under large combinatorial problems, such as the OPF problem, returning a good solution. The metaheuristics most used to solve OPF problems have been Genetic Algorithm (GA);
Particle Swarm Optimization (PSO); Simulated Annealing (SA); and Ant Colony Optimization (ACO).

These techniques are mainly used due to their competitive computational resources usage and good performances in large combinatorial problems. In this paper, an ACO is

proposed to solve the OPF problem of the Iraqi Super High Voltage Network (SHV) in an economic dispatch context.

\section{PROBLEM FORMULATION}

Optimal Power Flow is defined as the process of allocating generation levels to the thermal generating units in service within the power system, so that the system load is supplied entirely and most economically $[5,6]$. The objective of the OPF problem is to calculate, for a single period of time, the output power of every generating unit so that all demands are satisfied at minimum cost, while satisfying different technical constraints of the network and the generators. The problem can be modeled by a system which consists of $\mathbf{n g}$ generating units connected to a single bus-bar serving an electrical load Pd. The input to each unit shown as $\mathbf{F i}$, represents the generation cost of the unit. The output of each unit Pgi is the electrical power generated by that particular unit. The total cost of the system is the sum of the costs of each of the individual units.

The essential constraint on the operation is that the sum of the output powers must equal to the load demand. The standard OPF problem can be written in the following form:

$$
\left\{\begin{array}{c}
\min \{\mathrm{F}(\mathrm{x})\} \\
\text { Subject to }: \mathrm{h}(\mathrm{x})=0 \\
\text { and } \mathrm{g}(\mathrm{x}) \leq 0
\end{array}\right.
$$

where $\mathrm{F}(\mathrm{x})$ the objective function, $\mathrm{h}(\mathrm{x})$ represents the equality constraints, $\mathrm{g}(\mathrm{x})$ represents the inequality constraints and $\mathrm{x}$ is the vector of the control variables, that is those which can be varied by a control center operator (generated active and reactive powers, generation bus voltage magnitudes, transformers).

Generally, the OPF problem can be expressed as minimizing the cost of production of the real power which is given by objective function FT:

$$
\mathrm{F}_{\mathrm{T}}=\sum_{\mathrm{i}=1}^{\mathrm{ng}} \mathrm{F}_{\mathrm{i}}\left(\mathrm{P}_{\mathrm{gi}}\right)
$$

The fuel cost function of a generator is usually represented with a second-order polynomial:

$$
\mathrm{F}_{\mathrm{i}}=\sum_{\mathrm{i}=1}^{\mathrm{ng}}\left(\mathrm{a}_{\mathrm{i}}+\mathrm{b}_{\mathrm{i}} \mathrm{P}_{\mathrm{gi}}+\mathrm{c}_{\mathrm{i}} \mathrm{P}_{\mathrm{gi}}^{2}\right)
$$

Where $\mathbf{n g}$ is the number of generation including the slack bus. $\mathbf{P g}$ is the generated active power at bus $\mathbf{i}$. $\mathbf{a}_{\mathbf{i}}, \mathbf{b}_{\mathbf{i}}$ and $\mathbf{c}_{\mathbf{i}}$ are the unit costs curve for ith generator. 
The standard OPF problem can be described mathematically as an objective with two constraints as:

$$
\begin{array}{ll}
\sum_{i=1}^{\text {ng }} \mathrm{Pg}_{\mathrm{i}}=\mathrm{Pd}_{\mathrm{i}+} \mathrm{P}_{\mathrm{L}}, & \mathrm{i}=1: \mathrm{ng} \\
\mathrm{Pg}_{\mathrm{i}}^{\text {min }} \leq \mathrm{Pg}_{\mathrm{i}} \leq \mathrm{Pg}_{\mathrm{i}}^{\text {max }}, & \mathrm{i}=1: \mathrm{ng}
\end{array}
$$

where:

$\mathrm{Pg}_{\mathrm{i}}$ : Real power output of $\mathrm{i}^{\text {th }}$ generator (MW);

$\mathrm{F}_{\mathrm{i}}\left(\mathrm{Pg}_{\mathrm{i}}\right)$ : Operating cost of unit i $(\$ / \mathrm{h})$;

Pd: Total demand (MW);

PL: Transmission losses (MW);

$\mathrm{Pg}_{\mathrm{i}}^{\min }, \mathrm{Pg}_{\mathrm{i}}^{\max }$ : Operating power limits of unit $\mathrm{i}(\mathrm{MW})$;

$\mathrm{ng}$ : Total number of units in service.

\section{ANT COLONY OPTIMIZATION}

Ant colonies, and more generally social insect societies, are distributed systems that, in spite of the simplicity of their individuals, present a highly structured social organization. As a result of this organization, ant colonies can accomplish complex tasks that in some cases far exceed the individual capabilities of a single ant [7].

Ant colony optimization (ACO) is based on the cooperative behavior of real ant colonies, which are able to find the shortest path from their nest to a food source via a form of indirect communication. The method was developed by Dorigo and his associates in the early 1990s [8,9]. The ant colony optimization process can be explained by representing the optimization problem as a multilayered graph as shown in Fig. 1, where the number of layers is equal to the number of design variables and the number of nodes in a particular layer is equal to the number of discrete values permitted for the corresponding design variable[10].

Thus each node is associated with a permissible discrete value of a design variable. Figure 1 denotes a problem with four design variables with five permissible discrete values for each design variable.

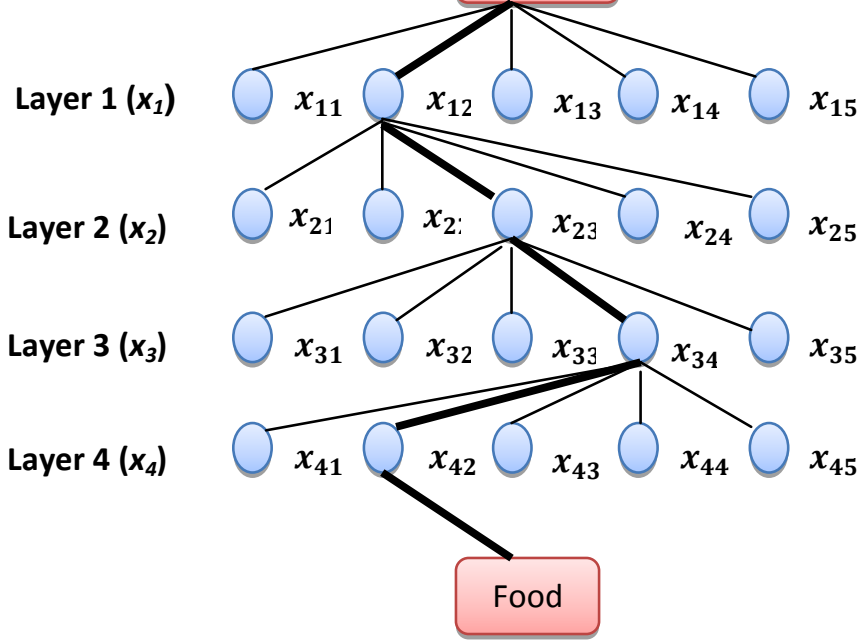

Figure (1) Graphical representation of the ACO process in the form of a multi-layered network

The ACO process can be explained as follows. Let the colony consist of $\mathbf{N}$ ants.
The ants start at the home node, travels through the various layers from the first layer to the last or final layer, and end at the destination node in each cycle or iteration. Each ant can select only one node in each layer, in accordance with the state transition rule given by Eq. (6). The nodes selected along the path visited by an ant represent a candidate solution. For example, a typical path visited by an ant is shown by thick lines in Fig. 1 This path represents the solution $\left(x_{12}, x_{23}, x_{34}\right.$, $x_{42}$ ). Once the path is complete, the ant deposits some pheromone on the path based on the local updating rule given by Eq. (7). When all the ants complete their paths, the pheromones on the globally best path are updated using the global updating rule described by Eqs. (7) and (8).

In the beginning of the optimization process (i.e., in iteration 1), all the edges or rays are initialized with an equal amount of pheromone. As such, in iteration 1, all the ants start from the home node and end at the destination node by randomly selecting a node in each layer. The optimization process is terminated if either the prespecified maximum number of iterations is reached or no better solution is found in a prespecified number of successive cycles or iterations. The values of the design variables denoted by the nodes on the path with largest amount of pheromone are considered as the components of the optimum solution vector. In general, at the optimum solution, all ants travel along the same best (converged) path.

\section{THE STATE TRANSITION RULE}

An ant k, when located at node $i$, uses the pheromone trail $\tau_{i j}$ to compute the probability of choosing $\mathrm{j}$ as the next node:

$$
P_{i j}^{(K)}=\left\{\begin{array}{cl}
\frac{\tau_{i j}^{\alpha}}{\sum_{j \in N_{i}^{(k)}} \tau_{i j}^{\alpha}} \quad \text { if } j \in N_{i}^{(k)} \\
0 \quad \text { if } j \notin N_{i}^{(k)}
\end{array}\right.
$$

where $\alpha$ denotes the degree of importance of the pheromones and $\mathrm{N}_{\mathrm{i}}^{(\mathrm{k})}$ indicates the set of neighborhood nodes of ant $\mathrm{k}$ when located at node $i$. The neighborhood of node $i$ contains all the nodes directly connected to node i except the predecessor node (i.e., the last node visited before i). This will prevent the ant from returning to the same node visited immediately before node i. An ant travels from node to node until it reaches the destination (food) node.

\section{PATH RETRACING AND PHEROMONE UPDATING}

Before returning to the home node (backward node), the kth ant deposits $\Delta \tau^{(\mathrm{k})}$ of pheromone on arcs it has visited. The pheromone value $\tau_{i j}$ on the arc $(i, j)$ traversed is updated as follows:

$$
\tau_{i j} \leftarrow \tau_{i j}+\Delta \tau^{(k)}
$$

Because of the increase in the pheromone, the probability of this arc being selected by the forthcoming ants will increase.

\section{PHEROMONE TRAIL EVAPORATION}

When an ant $\mathrm{k}$ moves to the next node, the pheromone evaporates from all the arcs $\mathrm{ij}$ according to the relation:

$$
\tau_{\mathrm{ij}} \leftarrow(1-\mathrm{P}) \tau_{\mathrm{ij}} ; \forall(\mathrm{i}, \mathrm{j}) \in \mathrm{A}
$$

Where $\mathrm{P} \in(0,1)$ is a parameter and $\mathrm{A}$ denotes the segments or arcs traveled by ant $\mathrm{k}$ in its path from home to destination. The decrease in pheromone intensity favors the exploration of different paths during the search process. This favors the elimination of poor choices made in the path selection. This also helps in bounding the maximum value attained by the 
pheromone trails. An iteration is a complete cycle involving ant's movement, pheromone evaporation and pheromone deposit.

After all the ants return to the home node (nest), the pheromone information is updated according to the relation

$$
\tau_{i j}=(1-\rho) \tau_{i j}+\sum_{k=1}^{N} \Delta \tau_{i j}^{(k)}
$$

Where $\rho \in(0,1)$ is the evaporation rate (also known as the pheromone decay factor) and $\Delta \tau_{\mathrm{ij}}^{(\mathrm{k})}$ is the amount of pheromone deposited on arc ij by the best ant k. The goal of pheromone update is to increase the pheromone value associated with good or promising paths. The pheromone deposited on arc ij by the best ant is taken as

$$
\Delta \tau_{i j}^{(\mathrm{k})}=\frac{\mathrm{Q}}{\mathrm{L}_{\mathrm{k}}}
$$

where $\mathrm{Q}$ is a constant and $\mathrm{L}_{\mathrm{k}}$ is the length of the path traveled by the kth ant (in the case of the travel from one city to another in a traveling salesman problem). Equation (10) can be implemented as

$$
\Delta \tau_{\mathrm{ij}}^{(\mathrm{k})}=\left\{\begin{array}{c}
\frac{\text { ffbest }}{\text { fworst } ;} \text { if }(\mathrm{i}, \mathrm{j}) \in \text { global best tour } \\
0 ; \text { otherwise }
\end{array}\right.
$$

where fworst is the worst value and fbest is the best value of the objective function among the paths taken by the $\mathrm{N}$ ants, and $\zeta$ is a parameter used to control the scale of the global updating of the pheromone. The larger the value of $\varsigma$, the more pheromone deposited on the global best path, and the better the exploitation ability. The aim of Eq. (7) is to provide a greater amount of pheromone to the tours (solutions) with better objective function values.

\section{ANT COLONY BASED OPF}

This section presents the formulation of the ACO algorithm adaptation to the OPF problem considered in this paper. The fitness function (f) used in the proposed methodology has been built by adding the equality constraint(4) to the total generation cost (2) .

$$
\mathrm{f}=\sum_{\mathrm{i}=1}^{\mathrm{ng}}\left(\mathrm{a}_{\mathrm{i}}+\mathrm{b}_{\mathrm{i}} \mathrm{P}_{\mathrm{gi}}+\mathrm{c}_{\mathrm{i}} \mathrm{P}_{\mathrm{gi}}^{2}\right)+\lambda\left(\sum_{\mathrm{i}=1}^{\mathrm{ng}} \mathrm{Pg}_{\mathrm{i}}-\sum_{\mathrm{i}=1}^{\mathrm{nb}} \mathrm{pd}_{\mathrm{i}}-\mathrm{P}_{\mathrm{L}}\right)^{2}
$$

The use of penalty functions in many OPF solutions techniques to handle inequality constraints can lead to convergence problem due to the distortion of the solution surface. In this method only the equality constraints (4) are used in the cost function. And the inequality constraints (5) are scheduled in the ACO process. Because the essence of this idea is that the constraints are partitioned in two types of constraints, active constraints are checked using the ACO-OPF procedure and the reactive constraints are updating using an efficient NewtonRaphson load flow procedure.

After the search goal is achieved, or an allowable generation is attained by the ACO algorithm. It is required to performing a load flow solution in order to make fine adjustments on the optimum values obtained from the ACO-OPF procedure. This will provide updated voltages, angles and transformer taps and active power that it should be given by the slack generator.

\section{ANT COLONY ALGORITHM}

The step-by-step procedure of ACO algorithm for solving the OPF problem can be summarized as follows :
Step 1:

Run a full ac power flow to obtain PL , sub the values of PL in the fitness function (Eq. 12).

\section{Step 2:}

Assume a suitable number of ants in the colony $(\mathrm{N})$. Assume a set of permissible discrete values for each of the ng design variables. Denote the permissible discrete values of the design variable $\mathrm{Pgi}$ as $\mathrm{Pg}_{\mathrm{il}}, \mathrm{Pg}_{\mathrm{i} 2}, \ldots, \mathrm{Pg}_{\text {ip }}(\mathrm{i}=1,2, \ldots, \mathrm{ng})$. Assume equal amounts of pheromone $\tau_{\mathrm{ij}}^{(1)}$ initially along all the arcs or rays (discrete values of design variables) of the multilayered graph shown in Fig. 1 The superscript to $\tau_{\mathrm{ij}}$ denotes the iteration number. For simplicity, $\tau_{\mathrm{ij}}^{(1)}=1$ can be assumed for all arcs ij. Set the iteration number $l=1$.

Step 3:

(a) Compute the probability $\left(\mathrm{P}_{\mathrm{ij}}\right)$ of selecting the arc or ray (or the discrete value) $\mathrm{Pg}_{\mathrm{ij}}$ as

$$
\mathrm{P}_{\mathrm{ij}}=\frac{\tau_{\mathrm{ij}}^{(l)}}{\sum_{\mathrm{m}=1}^{\mathrm{p}} \tau_{\mathrm{ij}}^{(l)}}
$$

which can be seen to be same as Eq. (6) with $\alpha=1$. A larger value can also be used for $\alpha$.

(b) The specific path (or discrete values) chosen by the kth ant can be determined using random numbers generated in the range $(0,1)$. For this, we find the cumulative probability ranges associated with different paths of Fig. 1 based on the probabilities given by Eq. (13). The specific path chosen by ant $\mathrm{k}$ will be determined using the roulette-wheel selection process in step 3(a).

Step 4:

(a) Generate $\mathrm{N}$ random numbers $\mathrm{r}_{1}, \mathrm{r}_{2}, \ldots, \mathrm{r}_{\mathrm{N}}$ in the range $(0$ 1 ), one for each ant. Determine the discrete value or path assumed by ant $\mathrm{k}$ for variable $\mathrm{i}$ as the one for which the cumulative probability range [found in step 2(b)] includes the value ri.

(b) Repeat step 3(a) for all design variables $\mathrm{i}=1,2, \ldots, \mathrm{ng}$.

(c) Evaluate the fitness function values corresponding to the complete paths (design vectors $\operatorname{Pg}(\mathrm{k})$ or values of Pgij chosen for all design variables $\mathrm{i}=1,2, \ldots$, ng by ant $\mathrm{k}, \mathrm{k}=1$, $2, \ldots, N)$ :

$$
\mathrm{f}_{\mathrm{k}}=\mathrm{f}\left(\mathrm{pg}^{(\mathrm{k})}\right) ; \mathrm{k}=1,2,3, \ldots \ldots, \mathrm{N}
$$

Determine the best and worst paths among the $\mathrm{N}$ paths chosen by different ants:

$$
\begin{aligned}
& \text { fbest }=\min \left\{\mathrm{f}_{\mathrm{k}}\right\} ; \mathrm{k}=1,2, \ldots, \mathrm{N} \\
& \text { fworst }=\max \left\{\mathrm{f}_{\mathrm{k}}\right\} ; \mathrm{k}=1,2, \ldots, \mathrm{N}
\end{aligned}
$$

Step 5:

Test for the convergence of the process. The process is assumed to have converged if all $\mathrm{N}$ ants take the same best path. If convergence is not achieved, assume that all the ants return home and start again in search of food. Set the new iteration number as $1=1+1$, and update the pheromones on different arcs (or discrete values of design variables) as

$$
\tau_{\mathrm{ij}}^{(\mathrm{l})}=\tau_{\mathrm{ij}}^{(\text {old })}+\sum_{\mathrm{k}} \Delta \tau_{\mathrm{ij}}^{(\mathrm{k})}
$$

Where $\tau_{\mathrm{ij}}^{\text {(old) }}$ denotes the pheromone amount of the previous iteration left after evaporation, which is taken as

$$
\tau_{\mathrm{ij}}^{(\mathrm{old})}=(1-\rho) \tau_{\mathrm{ij}}^{(l-1)}
$$


and $\Delta \tau_{\mathrm{ij}}^{(\mathrm{k})}$ is the pheromone deposited by the best ant $\mathrm{k}$ on its path and the summation extends over all the best ants $\mathrm{k}$ (if multiple ants take the same best path). Note that the best path involves only one arc ij (out of possible arcs) for the design variable $i$. The evaporation rate or pheromone decay factor $\rho$ is assumed to be in the range 0.5 to 0.85 and the pheromone deposited $\Delta \tau_{\mathrm{ij}}^{(\mathrm{k})}$ is computed using Eq. (3.6).

With the new values of $\tau_{\mathrm{ij}}^{(\mathrm{l})}$, go to step 3. Steps 3 , 4, and 5 are repeated until the process converges, that is, until all the ants choose the same best path i.e. values of Pgi that give the minimum cost, In some cases, the iterative process is stopped after completing a prespecified maximum number of iterations (lmax).

Step 6 :

Run a full ac power flow and provide updated voltages, angles and the new $\mathrm{Pg}_{1}$

\section{SYSTEM PARAMETERS AND COST FUNCTIONS}

The model of, 24 bus, $400 \mathrm{kV}(\mathrm{SHV})$ interconnected Iraqi power system is shown in Figure2.

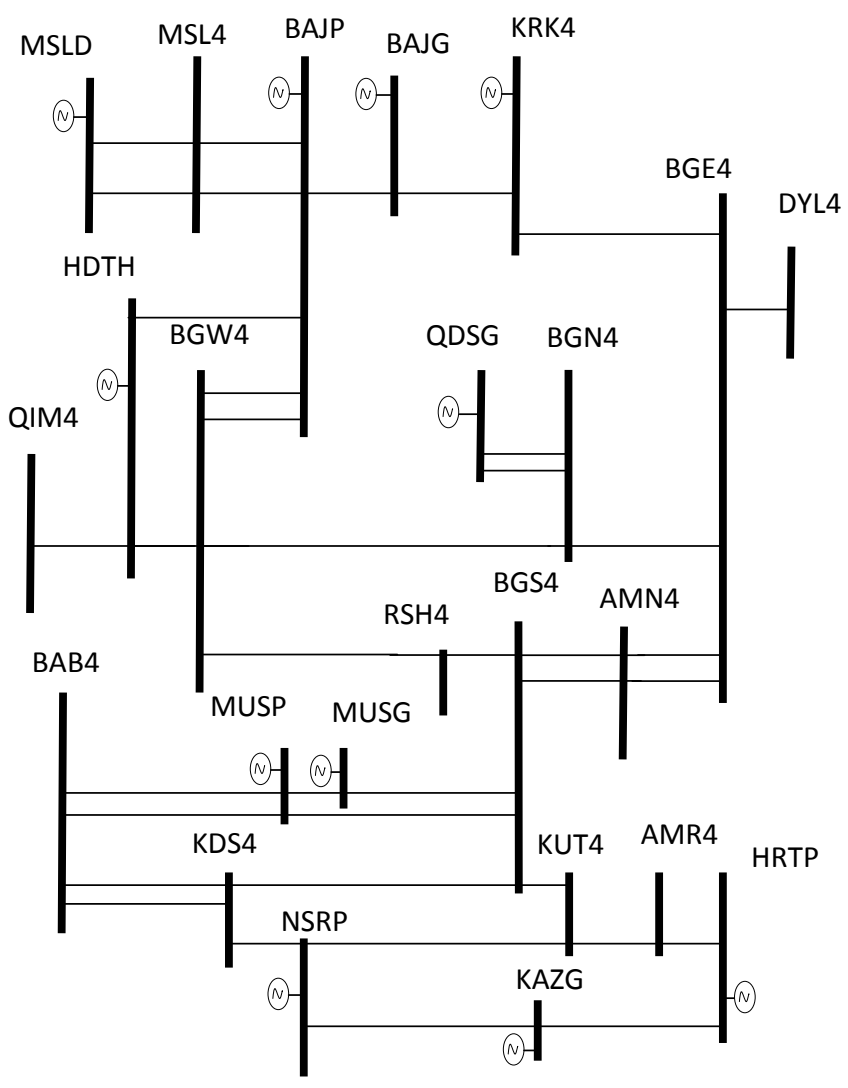

Fig. 2:The 24-bus 400-kV Interconnected Power System in Iraq

The bus codes and bus names are given in the Table 1 .
TABLE 1

THE BUS CODES AND BUS NAMES

\begin{tabular}{|c|c|c|c|c|c|}
\hline $\begin{array}{c}\text { Bus } \\
\text { name }\end{array}$ & $\begin{array}{c}\text { Bus } \\
\text { Code }\end{array}$ & $\begin{array}{c}\text { Bus } \\
\text { name }\end{array}$ & $\begin{array}{c}\text { Bus } \\
\text { Code }\end{array}$ & $\begin{array}{c}\text { Bus } \\
\text { name }\end{array}$ & $\begin{array}{c}\text { Bus } \\
\text { Code }\end{array}$ \\
\hline BAJP & 1 & NSRP & 9 & KSD4 & 17 \\
\hline BAJG & 2 & HRTP & 10 & BGN4 & 18 \\
\hline MSLD & 3 & KAZG & 11 & BGS4 & 19 \\
\hline KRK4 & 4 & MSL4 & 12 & AMN4 & 20 \\
\hline HDTH & 5 & QIM4 & 13 & KUT4 & 21 \\
\hline QDSG & 6 & BGW4 & 14 & AMR4 & 22 \\
\hline MUSG & 7 & BAB4 & 15 & BGE4 & 23 \\
\hline MUSP & 8 & RSH4 & 16 & DYL4 & 24 \\
\hline
\end{tabular}

The cost functions of the plants are constituted using the MS Excel Programming similarity curves. Cost functions and generation limits of the thermal plants are given in Table 2 [11], Note that plants three and five are hydro unit so that the fuel cost are zero.

TABLE 2

COST FUNCTIONS AND GENERATION LIMITS

\begin{tabular}{|c|c|c|}
\hline $\begin{array}{c}\text { Bus } \\
\text { Code }\end{array}$ & Cost Function $(\$ / \mathrm{h})$ & $\begin{array}{c}\text { Generators } \\
\text { Limits(MW) }\end{array}$ \\
\hline 1 & $0.05 P g_{1}^{2}+2.155 P g_{1}+2581$ & $120 \leq P g_{1} \leq 550$ \\
\hline 2 & $0.03 P g_{2}^{2}+11.91 P g_{2}+1698$ & $120 \leq P g_{2} \leq 450$ \\
\hline 3 & 0 & $130 \leq P g_{3} \leq 550$ \\
\hline 4 & $0.0136 P g_{4}^{2}+7.05 P g_{4}+154$ & $60 \leq P g_{4} \leq 250$ \\
\hline 5 & 0 & $60 \leq P g_{5} \leq 250$ \\
\hline 6 & $0.0017 P g_{6}^{2}+0.64 P g_{6}+200$ & $180 \leq P g_{6} \leq 600$ \\
\hline 7 & $0.154 P g_{7}^{2}+63.42 P g_{7}+517.3$ & $75 \leq P g_{7} \leq 250$ \\
\hline 8 & $0.0012 P g_{8}^{2}+0.35 P g_{8}+275$ & $200 \leq P g_{8} \leq 750$ \\
\hline 9 & $0.002 P g_{9}^{2}+0.561 P g_{9}+159$ & $120 \leq P g_{9} \leq 550$ \\
\hline 10 & $0.0025 P g_{10}^{2}+0.8 P g_{10}+120$ & $100 \leq P g_{10} 300$ \\
\hline 11 & $0.0158 P g_{11}^{2}+3.1 P g_{11}+658$ & $50 \leq P g_{11} \leq 250$ \\
\hline
\end{tabular}

\section{SIMULATION RESULTS}

The proposed approach has been applied on the Iraqi National Super High Voltage (SHV) grid. The potential and effectiveness of the proposed approach are demonstrated. The real data for Iraqi network have been taken from Iraq operation and control office.

Output power of the generator obtained from both the ant colony optimization (ACO) and the Linear Programming (LP) are given in Table 3.

TABLE 3

OUTPUT POWER OF THE GENERATOR AND LIMITED VALUES

\begin{tabular}{|c|c|c|c|c|}
\hline \multirow{2}{*}{ Bus } & \multirow{2}{*}{$\begin{array}{c}\text { Pgmin } \\
(\mathrm{MW})\end{array}$} & \multicolumn{2}{|c|}{ Pg (MW) } & \multirow{2}{*}{$\begin{array}{c}\text { Pgmax } \\
\text { (MW) }\end{array}$} \\
\hline 1 & 120 & 267.6 & 271.2 & 550 \\
\hline 2 & 120 & 285 & 281.66 & 450 \\
\hline 3 & 130 & 550 & 550 & 550 \\
\hline 4 & 60 & 250 & 250 & 250 \\
\hline 5 & 60 & 250 & 250 & 250 \\
\hline 6 & 180 & 600 & 600 & 600 \\
\hline 7 & 75 & 75 & 75 & 250 \\
\hline 8 & 200 & 750 & 750 & 750 \\
\hline 9 & 120 & 550 & 550 & 550 \\
\hline 10 & 100 & 300 & 300 & 300 \\
\hline 11 & 50 & 250 & 250 & 250 \\
\hline
\end{tabular}


As seen in Table 3, all of the output power of the generator is retained with these limits.

Table (4) shows the production cost, the active and reactive power loss when applying both Linear Programming method and ant colony optimization method.

\section{TABLE 4}

PRODUCTION COST, ACTIVE AND REACTIVE POWER LOSS

\begin{tabular}{|c|c|c|}
\hline & LP & ACO \\
\hline Total Cost (\$/h) & 30159 & 29670 \\
\hline MW Loss (MW) & 12.56 & 12.94 \\
\hline Mvar Loss (Mvar) & 110.62 & 113.72 \\
\hline
\end{tabular}

As seen in Table 4, the total generation cost in one hour $(29670 \$ / h)$ which obtained from the ant colony optimization is less than the cost obtained from the liner programming $(30159 \$ / \mathrm{h})$ bay an amount of $(489 \$ / \mathrm{h})$.

The bus voltage of each bus and limit values are shown in Table 5.

TABLE 5

THE BUS VOLTAGE AND LIMITS

\begin{tabular}{|c|c|c|c|c|}
\hline \multirow{2}{*}{ Bus } & \multirow{2}{*}{$\operatorname{Vmin}(p . u)$} & \multicolumn{2}{|c|}{$\mathrm{V}(\mathrm{p} . \mathrm{u})$} & \multirow{2}{*}{$\begin{array}{l}V_{\max } \\
\text { (p.u) }\end{array}$} \\
\hline & & LP & $\mathrm{ACO}$ & \\
\hline 1 & 0.95 & 0.997 & 1 & 1.05 \\
\hline 2 & 0.95 & 0.997 & 1 & 1.05 \\
\hline 3 & 0.95 & 1 & 1 & 1.05 \\
\hline 4 & 0.95 & 0.995 & 0.99 & 1.05 \\
\hline 5 & 0.95 & 0.995 & 1 & 1.05 \\
\hline 6 & 0.95 & 0.983 & 0.995 & 1.05 \\
\hline 7 & 0.95 & 0.984 & 1 & 1.05 \\
\hline 8 & 0.95 & 0.984 & 1 & 1.05 \\
\hline 9 & 0.95 & 0.970 & 0.970 & 1.05 \\
\hline 10 & 0.95 & 0.960 & 0.960 & 1.05 \\
\hline 11 & 0.95 & 0.960 & 0.960 & 1.05 \\
\hline 12 & 0.95 & 0.982 & 0.983 & 1.05 \\
\hline 13 & 0.95 & 0.988 & 0.994 & 1.05 \\
\hline 14 & 0.95 & 0.969 & 0.977 & 1.05 \\
\hline 15 & 0.95 & 0.978 & 0.992 & 1.05 \\
\hline 16 & 0.95 & 0.970 & 0.979 & 1.05 \\
\hline 17 & 0.95 & 0.972 & 0.984 & 1.05 \\
\hline 18 & 0.95 & 0.979 & 0.986 & 1.05 \\
\hline 19 & 0.95 & 0.977 & 0.988 & 1.05 \\
\hline 20 & 0.95 & 0.976 & 0.985 & 1.05 \\
\hline 21 & 0.95 & 0.967 & 0.973 & 1.05 \\
\hline 22 & 0.95 & 0.952 & 0.955 & 1.05 \\
\hline 23 & 0.95 & 0.976 & 0.984 & 1.05 \\
\hline 24 & 0.95 & 0.969 & 0.977 & 1.05 \\
\hline
\end{tabular}

As seen in Table 5, all of the bus voltages are retained with limits, and with ACO, the bus voltages are better than in LP with respect to the rated voltage.

Results of the OPF algorithm, bus angels, output reactive power of the each generator are shown in Table 6 .
TABLE 6

BUS ANGELS AND OUTPUT REACTIVE POWER OF THE GENERATORS

\begin{tabular}{|c|c|c|c|c|}
\hline \multirow{2}{*}{ Bus } & \multicolumn{2}{|c|}{ Bus Angle (deg) } & \multicolumn{2}{c|}{ Qgen (MVAR) } \\
\cline { 2 - 5 } & LP & ACO & LP & ACO \\
\hline 1 & 0 & 0 & 116.04 & 132.99 \\
\hline 2 & 0.024 & 0.0243 & -15.74 & 4.315 \\
\hline 3 & 2.370 & 2.39 & 222.53 & 207.26 \\
\hline 4 & -0.707 & -0.652 & 86.86 & 12.52 \\
\hline 5 & -1.137 & -1.144 & 9.2 & 7.766 \\
\hline 6 & -3.655 & -3.650 & 480 & 445.6 \\
\hline 7 & -3.017 & -3.096 & 34.75 & 106.5 .3 \\
\hline 8 & -2.959 & -3.038 & 346.9 & 404.5 \\
\hline 9 & -1.438 & -1.403 & 68.62 & 20.53 \\
\hline 10 & -1.785 & -1.723 & 103.46 & 94.64 \\
\hline 11 & -1.431 & -1.39 & 51.42 & 51.41 \\
\hline 12 & 0.583 & 0.599 & 0 & 0 \\
\hline 13 & -2.085 & -2.082 & 0 & 0 \\
\hline 14 & -4.210 & -4.197 & 0 & 0 \\
\hline 15 & -3.487 & -3.541 & 0 & 0 \\
\hline 16 & -4.264 & -4.261 & 0 & 0 \\
\hline 17 & -3.932 & -3.952 & 0 & 0 \\
\hline 18 & -3.879 & -3.873 & 0 & 0 \\
\hline 19 & -3.905 & -3.925 & 0 & 0 \\
\hline 20 & -4.069 & -4.073 & 0 & 0 \\
\hline 21 & -4.611 & -4.591 & 0 & 0 \\
\hline 22 & -4.613 & -4.575 & 0 & 0 \\
\hline 23 & -4.072 & -4.085 & 0 & 0 \\
\hline 24 & -4.559 & -4.548 & 0 & 0 \\
\hline & & & & \\
\hline
\end{tabular}

Figure (3) shows the performance of the Ant Colony optimization to solve the optimal power problem for the Iraqi SHV network.

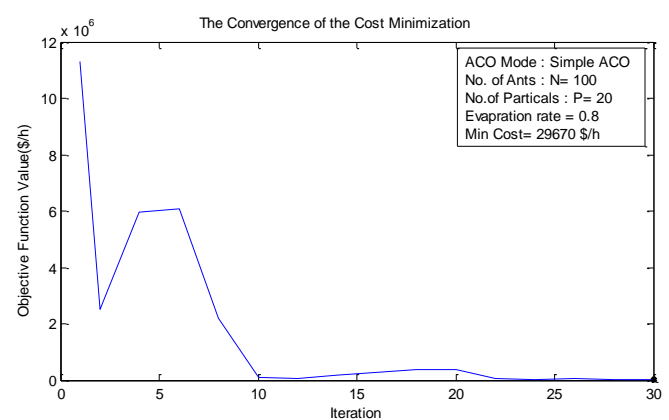

Fig. 3:The convergence of the cost minimization with respect to the number of iterations

\section{CONCLUSION}

The Ant Colony Optimization (ACO) is very successful in minimizing the generating cost, while maintaining system security. The ACO is very efficient than Linear Programming (LP) method because the Linear programming performance is dependent on the number of segments of the linearized generating cost function, while ACO does not to linearize the generating cost function. The ACO has an effective and robust performance for solving an Optimal Power Flow (OPF) problem. 
The advantages of the ACO approach are:

- Simple implementation

- Rapid discovery of good solutions

- Derivative free

- Very few algosrithm parameters

The convergence of a ACO is dependent on the number of design variables and the number of particles given for each design variable, If the control variables increased, the convergence would be slower, in another word convergence is guaranteed, but time to convergence uncertain.

\section{REFERENCES}

[1] J. Carpienter, "Contribution e l'étude do Dispatching Economique", Bulletin Society Française Electriciens, Vol. 3, August 1962.

[2] K. S. Pandya, S.K. Joshi, "A Survey of the Optimal Power Flow Methods", Journal of Theoretical and Applied Information Technology (JATIT), pp. 450-458, 2008.

[3] Z. F. Qiu, G. Deconnick and R. Belmans, "A Literature Survey of Optimal Power Flow Problems in the Electricity Market Context, " 2009 IEEE/Pes Power Systems Conference and Exposition, Vols 1-3, pp. 1845-1850, 2009

[4] J. A. Momoh, M. E. El-Hawary and R. Adapa, "A review of selected optimal power flow literature to 1993 part I: Non Linear and quadratic programming approaches, "
IEEE Transactions on Power Systems, vol. 14, pp. 96-104, Feb 1999.

[5] Wood A. J., Wollenberg B. F., "Power Generation, Operation and Control", New York, John Wiley and Sons, 1984.

[6] Chowdhury B. H., Rahman S., "A Review Of Recent Advances In Economic Dispatch", IEEE Transactions on Power Systems, Vol. 5, No. 4, pp. 1248-1259, 1990.

[7] M. Dorigo and T. Stuzel, "Ant Colony Optimization", The MIT Press, 2004

[8] A. Colorni, M. Dorigo, and V. Maniezzo,"Distributed optimization by ant colonies, in Proceedings of the First European Conference on Artificial Life", F. J. Varela and P. Bourgine, Eds., MIT Press, Cambridge, MA, pp. 134142, 1992.

[9] M. Dorigo, V. Maniezzo, and A. Colorni, "The ant system optimization by a colony of cooperating agents", IEEE Transactions on Systems, Man, and Cybernetics-Part B, Vol. 26, No. 1, pp. 29-41, 1996.

[10] S. Rao Singiresu, "Engineering Optimization Theory and Practice", John Wiley \& Sons, 2009.

[11] Montather Fadhil Meteb, "Particle Swarm Optimization (PSO) Based Optimal Power Flow For The Iraqi EHV Network",A thesis submitted to the university of Baghdad,2012. 